\title{
Asymptotic conditions of motion for radiating charged particles
}

\author{
James L. Anderson \\ Department of Physics, Stevens Institute of Technology, \\ Hoboken, New Jersey 07030
}

\begin{abstract}
Approximate asymptotic conditions on the motion of compact, electrically charged particles are derived within the framework of general relativity using the Einstein, Infeld, Hoffmann (EIH) surface integral method. While superficially similar to the Abraham-Lorentz and Lorentz-Dirac equations, these conditions differ from them in several fundamental ways. They are not equations of motion in the usual sense but rather a set of conditions which the these motions must obey asymptotically in the future of an initial starting time. And furthermore, they do not admit the run-away solutions of these other equations. As in the original EIH work, they are integrability conditions gotton from integrating the empty-space (i.e., sourceless) Einstein-Maxwell equations of general relativity over closed two-surfaces surrounding the sources of the fields appearing in these equations.. No additional ad hoc assumptions, such as the form of a force law or the introduction of inertial reaction terms, are required for this purpose. Nor is there a need for any infinite mass renormalizations such as are required in other derivations since all integrals are over surfaces and thus finite. In addition to being asymptotic, the conditions of motion derived here are also approximate and apply, as do the original EIH equations, only to slowly moving systems. A 'slowness' parameter $\epsilon$ is identified as the ratio of the light travel time across the system divided by a characteristic time, e.g., a period. Use is made of both the method of matched asymptotic expansions and the method of multiple time scales to obtain an asymptotic expansion in $\epsilon$ and the expansion is carried to sufficiently high order $\epsilon^{7}$ to obtain the lowest order radiation reaction terms. The resulting conditions of motion are shown to not allow run-away motions.
\end{abstract}

PACS numbers: 03.50D, 04.40, 04.20M

\section{INTRODUCTION}

One of the most profound and far reaching consequences of Einstein's general theory of relativity is the restrictions it places on the motion of the sources of the fields described by the theory. This is a unique feature of general relativity and is found in no other field theory. In electrodynamics, for example, one must postulate not only the form of the Lorentz force law but also the form of the inertial forces that appear in the equations of motion. Furthermore, these restrictions, which I have called conditions of motion, follow from the empty-space field equations of the theory without the need to introduce source terms into these equations. As a consequence, many of the well-known difficulties and inconsistencies associated with point source models are avoided in general relativity. And finally, these conditions of motion are sufficiently restrictive that they limit the possible trajectories of an N-body system of compact sources to a $6 \mathrm{~N}$-parameter family of curves. In the final analysis, the origin of these conditions can be traced back to the general covariance of the field equations of general relativity. [1]

Einstein and his co-workers Infeld and Hoffmann (EIH) first derived approximate conditions of motion for systems of slowly moving $(v / c \ll 1)$ compact gravitating sources up to what is now called the post-Newtonian approximation in 1939. 2] Two subsequent papers on the subject were published by Einstein and Infeld in 1939 and 1949. [3] The procedure used by EIH involved the evaluation of integrals of the field equations over surfaces surrounding the sources of the fields appearing in these equations. By using only surface integrals they were thus able to avoid the need to perform volume integrals over regions containing the sources. Even though EIH spoke of "point source" solutions it is clear that they were really refering to the form of the solutions in the region exterior to these sources so that their derivation was applicable to compact sources and black holes. While the EIH surface integrals were exact their evaluation required what has come to be called the slow motion approximation. In this approximation one assumes that the fields and source variables depend on time scales that are long compared to the light travel time across the system of sources. In a 1986 paper [4] (referred to hereafter as AI) I extended this idea by introducing multiple time scales into the theory and was thereby able to avoid the introduction of the fictitious dipole fields used by EIH. I also showed how one could make use of the method of matched asymptotic expansions to include the effects of radiation reaction into the conditions of motion. These conditions followed as integrability conditions directly from the emptyspace Einstein field equations and were applicable both to sources with and without strong internal gravity and black 
holes. Unfortunately, the EIH papers are referenced only in passing even in standard texts on general relativity [5] and hardly at all in the literature. And yet these papers contain what I would claim is one of Einstein's greatest contributions to physics and the only reliable method to date for deriving conditions of motion.

In this paper I have applied the EIH formalism together with the methods developed in AI to the case of electrically charged sources up to and including the order in which radiation reaction terms first appears. Past attempts to derive an expression for the electrodynamic radiation reaction force within the framework of Newtonian or special relativistic physics have been beset with a number of well-known difficulties. The best known of the equations, the AbrahamLorentz (AL) equation and its special relativistic generalization, the Lorentz-Dirac (LD) equation (collectively the ADL equations) have been the source of continued discussion in the scientific literature. [6] - 14]. All derivations of these equations have required one or more $a d$ hoc assumptions concerning the form of a force law, an inertial reaction force or an indeterminate integral. Many derivations also require some form of infinite mass renormalization and they all make an arbitrary choice of the retarded over the advanced potential in constructing the field of a moving charge. Thus Dirac was forced to assume the form of the indeterminant (in fact, infinite) integrals that appeared in his derivation.

But perhaps the most serious problem associated with the ADL equations is the existence of the famous (or infamous, depending on one's point of view) run-away solutions possessed by these equations. Their existence alone should have been sufficient evidence that there was something basically wrong with the whole enterprise. Dirac sought to eliminate these solutions by imposing the condition that in the asymptotic future all accelerations should be finite. This condition however leads to the phenomena of "pre-acceleration" with its attendant acausality. As a consequence, several authors have been led to propose alternative equations of motion for radiating charges. [15] Such ad hoc modifications, motivated by little more than the desire to avoid the run-away sloution problem can hardly be considered to be a satisfactory solution to the problem. But more, it is far from obvious nor has it been shown that any of these equations are compatible with the restrictions imposed by general relativity on the motion of sources of the electromagnetic field. And indeed, if one accepts general relativity as a fundamental theory, one has no alternative than to accept, as one of its consequences, the restrictions it imposes on the motion of sources of both the electromagnetic and gravitational fields.

The first derivation of conditions on the motion of electrically charged sources that did not involve an infinite mass renormalization and/or ad hoc assumptions of the type discussed above was that of Infeld and Wallace (IW). [16] This derivation applied the EIH procedure to the coupled Einstein-Maxwell equations and was thus able to avoid the difficulties of the other derivations. Strangly, this paper is almost never refered to in the literature. However, in spite of its superiority over other derivations, the IW derivation did not overcome all of the difficulties of the ALD equation. IW gave seperate derivations using half-advanced plus half-retarded and pure retarded potentials and argued that the former was more "natural" even though there was no radiation damping in this case! In their derivations IW focused on one charge, which they took to be momentarily at rest, in an external field. After obtaining their conditions of motion for it from EIH type surface integrals they then performed a Lorentz boost to obtain conditions for arbitrary velocities. Unfortunately, dynamical effects involving accelerations can be missed in this way and indeed, such effects are not present in the IW conditions. They also assumed, as did EIH, that they had derived equations of motion rather than asymptotic conditions and hence were unable to rule out run-away solutions. And finally, theirs was a formal expansion in powers of a parameter $\lambda$ which was in the end set equal to unity.

In what follows I will present the details of the derivation of the conditions imposed on the motion of the sources of electromagnetic and gravitational fields by applying the EIH method to the coupled Einstein-Maxwell equations of general relativity. In the next section I will discuss briefly earlied derivations of the radiation reaction force with emphasis on the Dirac derivation which is nearest in spirit to the EIH method so that one can see the essential differences in the two approaches. Section III will present a derivation of the EIH surface integrals and Section IV will outline the approximation procedures needed to evaluate these integrals. Section V contains the details of the derivation and the paper concludes with a brief summary.

\section{CLASSICAL DERIVATIONS AND THEIR PROBLEMS}

By classical I mean here derivations of the electromagnetic radiation reaction force that do not use the general theory of relativity. Most of these derivations attempt to calculate in one way or another a "self"-force of a charged particle acting on itself. The first derivations of this kind were given in 1903 by Abraham and 1904 by Lorentz. [17] These early derivations made use of a charge model consisting of a rigid, spherically symmetric charge distribution $\rho(\mathbf{x})$ of radius $a$ and calculated this force by summing up the interactions of pairs of the infinitesimal elements comprising the charge. Because of the assumption of rigidity the results of these calculations were only applicable to non-relativistic motions. The result of these calculations was a series expansion of the form 


$$
\mathbf{F}_{\text {self }}=\sum_{n=0}^{\infty} \mathbf{F}_{n}
$$

where

$$
\begin{gathered}
\mathbf{F}_{0}=-\frac{4}{3} \frac{U}{c^{2}} \dot{\mathbf{v}}, \\
\mathbf{F}_{1}=\frac{2}{3} \frac{e^{2}}{c^{3}} \ddot{\mathbf{v}}
\end{gathered}
$$

and

$$
\mathbf{F}_{n} \sim \frac{e^{2}}{n ! c^{n+2}} \stackrel{(n+1)}{\mathbf{v}} a^{n-1} .
$$

Here $\mathbf{v}$ is the velocity of the charge, $e$ its total charge and $U$ its so-called self-energy which is given by

$$
U=\frac{1}{2} \int d^{3} x \int d^{3} x^{\prime} \frac{\rho(x) \rho\left(x^{\prime}\right)}{\left|\mathbf{x}-\mathbf{x}^{\prime}\right|} .
$$

It is this term which gives most of the trouble in these derivations since it is proportional to $e^{2} / c^{2} a$. In order to eliminate the higher order terms in the expression for $\mathbf{F}_{\text {self }}$ one would like to take the limit $a \rightarrow \infty$. But then of course $U$ diverges. There is however, in my view, an even more serious problem with these derivations. In order to evaluate $\mathbf{F}_{\text {self }}$ one must make use of the Lorentz force law. But that use does not in and of itself justify inserting it into the right hand side of the Newtonian equations of motion, a procedure I would call proof by naming. Nor can one be sure that the form of Newton's inertial force, $m_{0} \dot{\mathbf{v}}$ or its special relativistic generalization, is valid when dealing with charged particles. If one does proceed in this manner, then one is forced to take for $m_{0}$, the expression

$$
m_{0}=m-\frac{4}{3} \frac{U}{c^{2}},
$$

where $m$ is the physical (finite) mass of the charge, in order to obtain a finite result in the limit $a \rightarrow \infty$. In this limit the bare mass $m_{0}$ is of course negatively infinite.

In order to avoid the non-relativistic restrictions of the AL model, Feynman [8] suggested using a cutoff procedure in which the Fourier representation of the retarded Green function is altered by making the substitution

$$
\frac{1}{k^{2}} \rightarrow \frac{1}{k^{2}}-\frac{1}{k^{2}-\Lambda^{2}}
$$

where $\Lambda$ is the cutoff factor and is ultimately allowed to become infinite. Using this modified Green function, one can calculate the self-force of a point charge on itself by evaluating the Lorentz force at the location of the charge. [9] The result is finite and is the relativistic extension of the AL self force with the charge radius $a$ replaced by $1 / \Lambda$.. Aside from this feature, this derivation suffers from the same defects of the AL derivation including the infinite mass renormalization.

A different approach was taken by Dirac [14 that has some of the features of the EIH derivation and is reviewed here so that the reader can compare the two derivations. Dirac considered point charges and defined what he called the electromagnetic radiation field $F_{\text {rad }}^{\mu \nu}$ to be

$$
F_{\text {rad }}^{\mu \nu}=F^{+\mu \nu}-F^{-\mu \nu}
$$

where $F^{+\mu \nu}$ and $F^{-\mu \nu}$ are respectively the retarded and advanced fields of a such a charge. While these latter fields are infinite on the charges trajectory, the radiation field is not and is given there by

$$
F_{\text {rad }}^{\mu \nu}=\frac{4 e}{3}\left(\dddot{z}^{\mu} \dot{z}^{\nu}-\dddot{z}^{\nu} \dot{z}^{\mu}\right) .
$$

where $z^{\mu}(\tau)$ are the coordinates of the charge expressed as a function of its proper time $\tau$ and where dots over a quantity indicate differentiation with respect to $\tau$. Dirac argued that his definition of the radiation field was physically reasonable since, at large distances from the charge and at the correspondingly large times after an acceleration takes place, the advanced field will be zero. He then assumed the existence of an energy-stress tensor 


$$
T^{\mu \nu}=T_{\mathrm{matter}}^{\mu \nu}+T_{\mathrm{em}}^{\mu \nu}
$$

where $T_{\text {matter }}^{\mu \nu}$ is a matter energy-stress tensor localized on the particles trajectory and

$$
T_{\mathrm{em}}^{\mu \nu}=\frac{1}{4 \pi}\left(\frac{1}{4} F_{\rho \sigma} F^{\rho \sigma} \eta^{\mu \nu}-F^{\rho \mu} F^{\sigma \nu} \eta_{\rho \sigma}\right)
$$

is the electromagnetic energy-stress tensor. In these equations $\eta_{\mu \nu}=\operatorname{diag}(1,-1,-1,-1)$ is the Minkowski metric tensor and $\eta^{\mu \nu}$ is its inverse, Greek indicies take the values $0, \ldots 3$, and the Einstein summation convention is used throughout. The total energy-stress tensor is assumed to be conserved so that

$$
T_{, \nu}^{\mu \nu}=0
$$

where the comma notation is used to denote differentiation, that is ${ }_{, \mu} \equiv \partial_{x^{\mu}}$.

Dirac proceeds by integrating this conservation law over the four-volume contained within a thin tube surrounding a piece of the charge's trajectory and converting the result into an integral over the surface of the tube by means of Gauss's theorem to obtain

$$
\left(\int_{\sigma_{1}}+\int_{\sigma_{2}}+\int_{S}\right) T^{\mu \nu} d S_{\nu}=0
$$

where $\sigma_{1}$ and $\sigma_{2}$ refer to the top and bottom caps respectively of the tube and $S$ refers to its wall. Since, by assumption, the support of the matter energy-stress tensor is the charge trajectory it does not contribute to the integral along the wall. On it Dirac evaluates $T_{\mathrm{em}}^{\mu \nu}$ using the total field $F_{\text {tot }}^{\mu \nu}$ given by

$$
F_{\text {tot }}^{\mu \nu}=F_{\text {in }}^{\mu \nu}+F^{+\mu \nu}
$$

where $F_{\text {in }}^{\mu \nu}$ is the field incident on the charge. The wall is such that its intersection with each space-like plane whose normal is parallel to $\dot{z}^{\mu}$ at the point of its intersection with the charge trajectory is a small sphere of radius $\varepsilon$. In order that this construction leads to a well-defined wall it is of course necessary that $\varepsilon$ be less than the curvature of the trajectory which is inversely proportional to the acceleration of the charge. Finally the field on each of these spheres is evaluated in terms of the coordinates of its instantaneous center. This evaluation cannot be given exactly since $F^{+\mu \nu}$ depends on the retarded position of the charge which is given by an expansion in $\varepsilon$ about its instantaneous position. The result of these calculations is that

$$
\int_{S} T_{\mathrm{em}}^{\mu \nu} d S_{\nu}=\int_{Q}^{Q^{\prime}}\left[\frac{1}{2} e^{2} \varepsilon^{-1} \ddot{z}^{\mu}-e \dot{z}^{\nu} f_{\nu}^{\mu}+\mathcal{O}(\varepsilon)\right] d \tau
$$

where $Q$ and $Q^{\prime}$ correspond to the points of the trajectory that intersect the top and bottom caps respectively and where

$$
f^{\mu \nu}=F_{\text {in }}^{\mu \nu}+\frac{1}{2}\left(F^{+\mu \nu}-F^{-\mu \nu}\right)
$$

The $\mathcal{O}(\varepsilon)$ term in $(2.15)$ is in fact an infinite series in $\varepsilon$ similar in form to the $F_{n}$ in the expression (2.1) above for $F_{\text {self }}$ which Dirac does not evaluate.

In order to complete the derivation it is of course necessary to evaluate the cap contributions in (2.13). But here one encounters two serious difficulties: one does not know $T_{\text {matter }}^{\mu \nu}$ initio and worse, the contribution to the caps from $T_{\mathrm{em}}^{\mu \nu}$ is divergent. As a consequence Dirac was forced to assume the form of the contributions from these caps. Since the final answer in any case must be independent of $\varepsilon$ these contributions must cancel the $\varepsilon$ dependent terms in the integral in 2.15) and not, as Dirac asserts, so that the integrals have a definite form in the limit $\varepsilon \rightarrow 0$. There is, in fact, no need to take this limit nor would it make any mathematical sense to do so. These contributions must also be consistent with the requirement, which follows from (2.13), that the line integral in (2.15) depend only on the end points $Q$ and $Q^{\prime}$ and hence the integrand in this integral be a perfect differential. Neglecting the $\varepsilon$ dependent terms which in all cases must cancel with contributions from the caps this latter condition tells us that

$$
e \dot{z}^{\nu} f_{\nu}^{\mu}=\dot{B}^{\mu}
$$

where $B^{\mu}$ is undetermined except for the requirement that $\dot{z}_{\mu} \dot{B}^{\mu}=0$ which follows from the antisymmetry of $f \mu \nu$ in $\mu$ and $\nu$. The simplest assumption that satisfies this requirement is that $B^{\mu}=m \dot{z}^{\mu}$ although, as Dirac pointed out, there are other possibilities. With this assumptionn the equations of motion become 


$$
m \ddot{z}^{\mu}=\frac{2}{3} e^{2}\left[\dddot{z}^{\mu}+\ddot{z}^{\nu} \ddot{z}_{\nu} \dot{z}^{\mu}\right]+e \dot{z}_{\nu} F_{\text {in }}^{\mu \nu}
$$

which Dirac took to be exact. However, buried within the assumption for $B^{\mu}$ is some kind of infinite mass renormalization to compensate for the divergent self-energy contribution of $T_{\mathrm{em}}^{\mu \nu}$ to the cap integration. If, for example, one took, for the matter energy-stress tensor the Minkowski tensor

$$
T_{\text {matter }}^{\mu \nu}=m_{0} \int \delta^{4}(x-z(\tau)) \dot{z}^{\mu} \dot{z}^{\nu} d \tau
$$

where $\delta^{4}(x)$ is the four-dimensional Dirac $\delta$-function, the bare mass $m_{0}$ would contain a negative infinite component similar to the $m_{0}$ in (2.6). As the reader will see in what follows, the EIH surface integral method circumvents this problem by in effect replacing the volume integrals in the cap contributions by surface integrals which are all finite. Finally, this equation, like its counterparts obtained from the use of one or the other radiation reaction force, possesses run-away solutions, that is, solutions for which the accelerations increases exponentially with time.

\section{EIH SURFACE INTEGRALS}

The derivation of the conditions of motion makes use of the fact that the Einstein-Maxwell field equations for the gravitational field $g_{\mu \nu}$ and the electromagnetic field $F^{\mu \nu}$ contain three dimensional curls. Therefore, when these equations are integrated over any closed 2-surface these curl terms vanish. When in particular such a surface encloses a source of these fields the requirement that the remaining surface terms must be surface independent and vanish leads to conditions on the motion of this source. To facilitate the construction of these surface integrals it is useful to write the Einstein-Maxwell field equations in the form (I use units in which $G=c=1$, Latin indices run from 1 to 3 and Greek indices from 0 to 3.)

$$
U^{\mu \nu \rho}{ }_{, \rho}=\Theta^{\mu \nu}
$$

where

$$
\begin{gathered}
U^{\mu \nu \rho}=-U^{\mu \rho \nu}=\frac{1}{16 \pi}\left\{(-g)\left(g^{\mu \nu} g^{\rho \sigma}-g^{\mu \rho} g^{\nu \sigma}\right)\right\}, \sigma \\
\Theta^{\mu \nu}=(-g)\left(T_{\mathrm{em}}^{\mu \nu}+t_{L L}^{\mu \nu}\right), \\
\left(\sqrt{-g} F^{\mu \nu}\right)_{, \nu}=0,
\end{gathered}
$$

and

$$
F[\mu \nu, \rho]=0 .
$$

Here $g=\operatorname{det}\left(g_{\mu \nu}\right), t_{L L}^{\mu \nu}$ is the Landau-Lifshitz pseudotensor [19], $T_{\mathrm{em}}^{\mu \nu}$ is the electromagnetic energy-stress tensor given by (2.11) (in what follows I will omit the subscript em to simplify the notation), indices are raised and lowered using $g_{\mu \nu}$ in the usual manner and brackets around a set of indices indicates that the indices are to be antisymmetrized.

Because of the antisymmetry of $F^{\mu \nu}$ in $\mu$ and $\nu$ and $U^{\mu \nu \rho}$ in $\nu$ and $\rho$, it follows that $F_{, s}^{r s}$ and $U_{, s}^{\mu r s}$ are three dimensional curls. As a consequence, integration of (3.1) over a closed spatial 2-surface in a $t=$ const. hypersurface gives the result

$$
\oint\left(U^{\mu r 0}{ }_{0}-\Theta^{\mu r}\right) n_{r} d S=0
$$

where $n_{r}$ is an outward pointing unit surface normal. Similarly, we get from equation (2.4)

$$
\oint\left(\sqrt{-g} F^{r 0}\right)_{, 0} n_{r} d S=0 .
$$

It is these last two equations that are used to obtain conditions of motion for our charged system. They come from noting that, since these equations must hold on any two-surface, the sums of the contributions to the integrals that are surface independent must by themselves be zero. (The surface dependent terms must in all cases vanish 
identically.) In practice one chooses the surfaces to be spheres centered on the sources and sets to zero those terms that do not vanish identically. (If there are no sources, so that the fields are divergence free inside the surfaces, then all the integrals vanish directly as a consequence of the field equations.)

Because one employs in the derivation only surface integrals on which the fields are everywhere finite, it follows that there are no infinite integrals that must be renormalized away. If we compare (3.6) with (2.13) we see that the divergent cap terms in the latter equation get replaced by the finite surface integrals of $U^{\mu r 0}{ }_{, 0}$ and $(-g) t_{L L}^{\mu \nu}$. It is the first of these integrals in fact that contribute the inertial force terms to the conditions of motion, obviating the need to postualte them. Furthermore, since the source region is contained entirely within the 2 -surfaces of integration in (3.6) and (3.7), one need only know the fields on these surfaces and not inside. All that is required is that each surface contain only one source, that their radii are sufficiently large that the fields on them are weak and small compared to the distance between sources. Thus one can equally well deal with compact sources with strong internal gravity, e.g., neutron stars and even black holes as with sources whose fields are weak everywhere. In particular, the problems associated with the use of point sources discussed in Section II do no occur in the EIH procedure.

\section{APPROXIMATION PROCEDURES}

The evaluation of the surface integrals in (3.6) and (3.7) requires a knowledge of the fields on these surfaces and this can only be gotten by employing some kind of perturbation scheme. The use of any such scheme requires that there be one or more small parameters associated with the system. In the present work one of these is a dimensionless 'slowness' parameter $\epsilon$ that is of the order of the ratio of the light travel time across the system of sources to a time scale associated with their motion, e.g. an orbital period. It is also of the order of the ratio of the size of the system to the wavelength of the radiation emitted by it and also the ratio of the velocities of the sources to the velocity of light. Its precise value is fixed by the parameters that characterize the source system and its motion. It should be emphasized that this parameter is not an arbitrary small parameter as EIH took it to be nor can it be taken to be the inverse of the velocity of light as many authors have done. In addition to assuming that $\epsilon$ is small compared to unity, I will assume that the relative strengths of the gravitational and electromagnetic interactions $\delta=$ mass/charge is small compared to unity and also to $\epsilon$ for all charges in the system. In this work I have only kept terms of order $\delta^{2}$ and I have also scaled masses and charges so that $m_{A}=\mathcal{O}\left(\delta^{2} \epsilon^{2}\right)$ and $q_{A}=\mathcal{O}\left(\delta \epsilon^{2}\right)$. These restrictions have the effect that the operation of raising and lowering indices on all tensorial quantities is accomplished with the help of the flat-space Minkowski tensor.

\section{A. Matched Asymptotic Expansions}

The perturbation methods employed here are the singular perturbation techniques used in AI to derive approximate conditions of motion for gravitationally interacting uncharged particles. The first of these is the method of matched asymptotic expansions which was first used by Laplace in 1805 (see Ref. [18] for this and other references to this method.) In general this method seeks to develop approximate solutions to a system of equations in different regions of the range of the independent variables using expansions appropriate to each region The two expansions are then 'matched' in an overlap region where both expansions are valid. In order to obtain approximate solutions to the Einstein-Maxwell equations it is in principle necessary to use four overlapping regions in all corresponding to two different matchings, one in time and one in space.

The time matching treats the region in the vicinity of an initial value surface as a boundary layer called here the fast zone where the solution depends on the initial value data. The solution in this region is matched to an asymptotic region in the future of the initial data surface $t=$ const. called the slow zone. The undetermined parameters and functions appearing in the solution in this latter region are then determined in terms of the initial value data by matching to the fast zone. The slow zone can be thought of as the region in which all initial transients generated by the initial data have died off.

The other matching is the one used by Burke. [20] It matches solutions in two overlapping spatial regions consisting of an inner or induction zone and an outer or radiation zone. What is important to us is that, in the lowest order of approximation the solution in the outer, slow zone is a pure outgoing solution, that is, a function of a null coordinate $u$ and the spatial coordinates $\mathbf{r}$. (In higher orders of approximation this region will contain both outgoing and incoming radiation due to backscattering.) It should be emphasized that this form of the solution is not imposed arbitrarily as it usually is in other treatments. Rather, it is a consequence of the fact that solutions of the wave equation have the property that so long as the energy of the field on the initial-value surface is finite then in the asymptotic future the field is purely outgoing. 21] 
As we shall see, it is the matching to this outgoing solution that is responsible for the phenomena of radiation damping and the appearance of irreversible terms in the conditions of motion to be derived here. Thus we see that irreversibility in the conditions of motion is a consequence of the finite energy condition imposed on the initial fields and not, as IW contended, because of an arbitrary choice in the use of retarded or advanced potentials. One could choose initial conditions on the fields that would lead to anti-damping for all times but this would violate the finite energy condition mentioned above. While the final result of the two approaches appears to be the same, the one used here is in accord with those used in other areas of physics where irreversibility arises in the asymptotic future as a consequence of restrictions on initial conditions. [22]

Although in principle one could construct solutions in the fast-time zone to obtain conditions of motion in this zone, I have not explicitly done so in this work. In general, the motion of the sources in the fast-time zone will depend in a very complicated way on the initial values not only of the source coordinates but those of the fields as well and will almost certainly not satisfy any unique set of equations of motion. Without this matching the conditions on the source motion obtained by the other matchings will then only be valid in the asymptotic future and are therefore not equations of motion in the usually accepted Newtonian sense. In particular, they cannot be used to solve initial value problems. I will defer a discussion of the implications of this state of affairs until after I outline a derivation of the conditions of motion accurate to radiation damping order.

\section{B. Multiple Time Scales}

In order to construct approximate expansions in the two spatial domains in the slow-motion domain it is necessary to make use of the method of multiple time scales. In their original work EIH employed only a rudimentary multiple time scale formalism. As a consequence they were forced to introduced fictitious dipole terms at each order of their expansion in $\lambda$ and in the end require that the sum of all these dipoles vanish. This procedure appeared somewhat arbitrary and may have cast some doubt on the validity of their results. In fact, when the multiple time scale formalism is used no such dipole terms are needed.

As the name implies, the multiple time scale formalism is used when one has to deal with systems in which phenomena occur on different time scales, e.g., oscillation and damping. Not only is it useful in separating out these different types of motion but it is used extensively to avoid the appearance of secular growth and nonuniformities in various approximation schemes. In its application one assumes that the dependent variables of a system of equations depend on the time variable $t$ via a sequence of times, $t_{0}, t_{1}, \ldots$ where usually, but not always, $t_{0}=t$ and $t_{n}=\epsilon^{n} t$, $\epsilon$ being a small dimensionless parameter in the problem. In this case, which is the one that applies in this work, a derivative with respect to $t$ is replaced by

$$
\partial_{t} \rightarrow \sum_{n=0} \epsilon^{n} \partial_{t_{n}}
$$

The dependence on these times is then fixed by the requirement that the terms responsible for secular growth vanish. (See AI and Ref. [18] for simple examples of the application of this method.)

The time scales required in general relativity will depend upon the problem being considered. If we are dealing with uncharged particles and if $t$ is the fastest time, characterized by the light crossing time of the system, then $\epsilon t$ will characterize the Newtonian time scale, $\epsilon^{3} t$ the post-Newtonian effects such as perihelion advance, $\epsilon^{5} t$ the post-post-Newtonian effects etc.. Gravitational radiation is characterized by $\epsilon^{6} t$, sometimes referred to as the $2 \frac{1}{2}$ post-Newtonian time scale (see Reference 11 for details.) When one is dealing with charged sources there are at least two different small parameters that characterize the system, $\epsilon$ and $\delta=m / q$, and hence the possibility of additional time scales arises. However. I have assumed that $\delta \ll \epsilon \ll 1$ so as to avoid having to deal with both electromagnetic and gravitational effects simultaneously. Consequently, $t_{1}=\epsilon t$ will characterize the Coulombian interaction time scale, $t_{3}=\epsilon^{3} t$ the Amperian or post-Coulombian time scale and radiation effects will be characterized by a $t_{4}=\epsilon^{4} t$ time scale in lowest order. 23]

\section{CONDITIONS OF MOTION}

The construction of approximate solutions of the field equations (3.1) and (3.4) and their use in evaluating the integrals in (3.6) and (3.7) follows the pattern developed in AI. (Here I will use the vector 4-potential $A_{\mu}$ where $F_{\mu \nu}=A_{\nu, \mu}-A_{\mu, \nu}$. As in AI, I assume the existence of a coordinate map such that $\mathfrak{g}=\sqrt{-g} g^{\mu \nu}$ can be expanded up to the order of accuracy needed here in an asymptotic series of the form 


$$
\mathfrak{g}^{\mu \nu} \sim \eta^{\mu \nu}+\sum_{n, m} \epsilon^{n} \delta^{m} h_{n m}^{\mu \nu}\left(\mathbf{r}, \mathbf{x}_{A}, q_{A}, m_{A}\right)
$$

where I have assumed that the $h_{n m}^{\mu \nu}$ depend on the multiple times through their dependence on the source coordinates $\mathbf{x}_{A}\left(t_{1}, t_{3}, \ldots, \epsilon, \delta\right)$, charges $q_{A}\left(t_{1}, t_{3}, \ldots, \epsilon, \delta\right)$ and masses $m_{A}\left(t_{1}, t_{3}, \ldots, \epsilon, \delta\right)$ with $A$ labeling the sources. One should keep in mind that $\mathfrak{g}^{\mu \nu}$ cannot be expanded indefinitely in a simple power series since it is not analytic in $\epsilon$, reflecting the fact that one encounters $\epsilon^{n} \ln (\epsilon)$ terms in higher orders of the approximation. 24. However, these terms do not appear at the level of accuracy used in this work. In a like manner I assume that the 4 -potential $A^{\mu}$ can be expanded as

$$
A^{\mu} \sim \sum_{n, m} \epsilon^{n} \delta^{m} A_{n m}^{\mu}\left(\mathbf{r}, \mathbf{x}_{A}, q_{A}, m_{A}\right)
$$

(Recall that, because of the smallness of $\delta$, indices are raised and lowered here using the Minkowski tensor so that, e.g., $A^{\mu}=\eta^{\mu \nu} A_{\nu}$.)

Because of the scalings chosen for our sources it follows that the expansion (5.1) starts with $\mathcal{O}\left(\delta^{2}\right)$ terms while the expansion (5.2) starts with $\mathcal{O}(\delta)$ terms. Since one only needs terms of this order to obtain an $\mathcal{O}\left(\delta^{2}\right)$ overall accuracy in the conditions of motion I will drop the index $m$ in the above expansions in what follows. Also, in this order the dependence of the source variables $\mathbf{x}_{A}, q_{A}, m_{A}$ on $\delta$ can be ignored so that from this point on I will ignore entirely all $\delta$ dependencies. Then, again because of the scaling employed, the lowest order in $\epsilon$ fields are $A_{2}^{0}$ and $h_{2}^{00}$.

In addition to the field expansions (5.1) and (5.2), it will also necessary to make certain assumptions concerning the dependence of the source variables $q_{A}, m_{A}$, and $\mathbf{x}_{A}$ on $\epsilon$. To the level of accuracy needed here I will assume that the latter two variables can be expanded as an asymptotic series in powers of $\epsilon$ of the form

$$
m_{A} \sim \epsilon^{2} m_{A 2}+\epsilon^{4} m_{A 4}+\cdots
$$

and

$$
\mathbf{x}_{A} \sim \mathbf{x}_{A 0}+\epsilon^{2} \mathbf{x}_{A 2}+\cdots
$$

where the dots indicate terms that are at least an order of magnitude in $\epsilon$ smaller that the terms retained. It should be emphasized that the powers of $\epsilon$ used in this expansion is not arbitrary but dictated by approximation itself. As we shall see, the masses $m_{A}$ will be automatically expanded as we proceed in the approximation while the charges $q_{A}$ are absolute constants of the motion and that there is no need to expand them at all.

To obtain equations for the fields $h_{n}^{\mu \nu}$ and $A^{\mu}$ from (3.1) and (3.4) I will impose the deDonder coordinate conditions

$$
\mathfrak{g}_{, \nu}^{\mu \nu}=0
$$

and the Lorentz gauge condition

$$
\mathrm{A}_{, \mu}^{\mu}=0
$$

Here too problems arise with the use of these conditions in higher orders of the approximation and they must be modified to eliminate the appearance $\ln (r)$ terms in these higher orders. 24]

At this point a difficulty arises. Usually when one substitutes an expansion of the dependent variables in powers of a small parameter into a system of equations one simply equates to zero the coefficients of the various powers of this small parameter. However, in the present case the coefficients $h_{n}^{\mu \nu}$ and $A_{n}^{\mu}$ in the expansions (5.1) and (5.2) themselves depend on $\epsilon$ through the dependence of the source variables on $\epsilon$. Therefore one cannot simply equate to zero the coefficients of the powers of $\epsilon$ when these expansions are substituted into the field equations. This difficulty seems to have been overlooked in all previous derivations of equations of motion in general relativity including AI as far as I know. It can however be dealt with in the following manner: Substitute the expansions (5.1) and (5.2) into the field equations and set $\epsilon=0$. It then follows from (3.1) that in the source-free weak-field region $\left.h_{2}^{00}\right|_{\epsilon=0}$ satisfies

$$
\left.\nabla^{2} h_{2}^{00}\right|_{\epsilon=0}=0
$$

However, since $h_{2}^{00}$ depends on $\epsilon$ solely through its dependence on the source variables it follows that it must satisfy (5.7) for all values of $\epsilon \ll 1$. Since at each stage of the approximation the unknown field coefficients in the expansions (5.1) and (5.2) enter the field equations linearly it follows that this argument holds quite generally and so allows us to proceed to find these coefficients without regard to the dependence of the source positions on $\epsilon$. 
The requirement that the sources are characterized only by their charge and mass is equivalent to requiring that, in the absence of other sources, the field of each source must be spherically symmetric. It follows therefore that $h_{2}^{00}$ is given by

$$
h_{2}^{00}=4 \sum m_{A 2} / r_{A}
$$

where the sum is over all sources in the system, i.e., over $A=1$ to $N, r_{A}^{i}=r^{i}-x_{A}^{i}$ where $x_{A}^{i}$ is the $i$ th coordinate of the Ath source and where, for convenience, we will take the center of mass of our system to be at the origin of coordinates so that $\sum m_{A} x_{A}^{i}=0$. (In the case of a charged black hole, $x_{A}^{i}$ is the effective coordinate of its center as determined by its exterior vacuum solution.) In a like manner the field equations (3.4) allow us to conclude that in the source-free region $A_{2}^{0}$ satisfies

$$
\nabla^{2} A_{2}^{0}=0
$$

and so is given, for spherically symmetric sources, by

$$
A_{2}^{0}=\sum q_{A} / r_{A}
$$

It cannot be emphasized too strongly that these solutions hold only in the exterior of the field sources and are not in any sense 'singular'. There are in fact no singular fields in the usual sense in general relativity as there are in special relativistic theories for example. The sources can be either compact matter sources or black holes which are modeled by our assumption that they have no internal dynamics and are characterized solely by their mass and charge. The fields (5.8) and (5.10) are exterior fields and by Birkhoff's theorem are unique.

It should also be explained why the coordinate $r_{A}$ appears in the above solutions and not it's zeroth approximation. Indeed, if we were to substitute the expansion (5.4) into these solutions we could then expand them about $\epsilon=0$, retain in them only the lowest order terms and add the remaining higher order terms to the higher order terms in the expansions (5.1) and (5.2). In effect, by proceeding in this manner we have effectively summed partial series that would have resulted had we not done so. By keeping all of these terms in the lowest order solutions (and we will continue this practice with all of the higher order terms) we accomplish two things: Firstly, it greatly simplifies the calculation due to the effective summing of numerous partial series. But secondly, and most importantly it enables us to center the surfaces in (3.6) and (3.7) on the true centers of the sources rather than on their approximate centers. Although of course one can use any surfaces one likes in these equations it again greatly simplifies the resulting calculations to use the true source centers since doing otherwise results in spurious multipoles appearing in the higher order approximations.

\section{A. Coulombian conditions of motion}

With the help of the fields $h_{2}^{00}$ and $A_{2}^{0}$ found above we are now able to determine the time dependence of the charges and masses appearing in the fields given above. To do so one needs to evaluate the surface integrals that appear in (3.6) with $\mu=0$ and in (3.7) respectively using these approximate solutions. For convenience one chooses spherical surfaces centered on the sources with radii large compared to their physical or Schwarzschild radii in the case of black holes but small compared to the distance between them. These restrictions on the size of these surfaces are necessary to insure that the weak field approximation employed here is valid in their neighborhoods. In carrying out these evaluations one finds two types of terms - those that depend on the radii of the surfaces and those that don't. Since the integrals as a whole must be independent of these radii it follows that the overall coefficients of the various powers of the radii must vanish identically. It also follows that the radii independent terms must also vanish since the integral as a whole must vanish. However, these terms will not vanish identically. The requirement that they do vanish imposes conditions on the motion of the sources as EIH showed. As a consequence, one need only pick out those terms in the integrands that are proportional to the inverse squares of the surface radii and direction independent and set their coefficients equal to zero to obtain these conditions.

Substitute then the expansion (5.1) into (3.6), divide by $\epsilon^{2}$ and set $\epsilon$ equal to zero. In this way one finds that

$$
\partial_{t_{1}} m_{A 2}=0 .
$$

The surface integral in equation (3.7) can be evaluated using $A_{2}^{0}$ given by $(5.10)$ without the need to set $\epsilon$ equal to zero since it is linear in $A^{0}$ and since higher order terms in the expansion (5.2) will not contribute to the integral because of their angular dependence. As a consequence we obtain the result that 


$$
\partial_{t} q_{A}=0
$$

The $q_{A}$ are thus absolute constants of the motion. Note that a similar argument does not pertain in the case of the masses because, in higher orders of the approximation, $\Theta^{0 r}$ in (3.6) will in general be non-zero.

The next quantity needed to obtain the lowest order conditions of motion is the lowest order contribution to the field $h^{0 r}$. In order to satisfy the deDonder conditions 5.5 we see that these contributions must be of order $\epsilon^{3}$ since $h_{2}^{00}{ }_{, 0}$ is of this order and differentiation with respect to a spatial coordinate does not change the order of a quantity. It then follows from the field equations and the deDonder conditions, using reasoning similar to that which led to the solution (5.8), that $h_{3}^{0 r}$ satisfies

$$
\nabla^{2} h_{3}^{0 r}=0
$$

and therefore is given by

$$
h_{3}^{0 r}=4 \sum m_{A 2} \dot{x}_{A r} / r_{A}
$$

where a dot over a quantity denotes differentiation with respect to $t_{1}$.

Equation (3.6) with $\mu=s$ can now be used in conjunction with the above fields to obtain the lowest, Coulombian conditions of motion. For this purpose we need to know $T^{s r}$ to $\mathcal{O}\left(\epsilon^{4}\right)$ and $U^{s r 0}$ to $\mathcal{O}\left(\epsilon^{3}\right)$. The integral over the spherical surface surrounding the $A$ th source appearing in (3.6) will vanish provided the overall coefficient of the terms in the integrand proportional to $1 / r_{A}^{2}$ that remain after dividing by $\epsilon^{4}$ and setting $\epsilon$ equal to zero vanishes. This will be the case if

$$
m_{A 2} \ddot{x}_{A 0}^{s}=q_{A} F_{2 A}^{s 0}
$$

where $F_{2 A}^{s 0}$ is the lowest order electric field at the location of the $A$ th source due to all the other sources and is given by

$$
F_{2 A}^{s 0}=\sum^{\prime} q_{B} \frac{x_{A B 0}^{s}}{x_{A B 0}^{3}}
$$

where $x_{A B}^{s}=x_{A}^{s}-x_{B}^{s}$ and the prime on the sum indicates that it is over all $B \neq A$. Note that, at this order of approximation, our conditions of motion involve only the lowest order contributions to the masses and positions of the sources. However, unlike in the case of the fields, we cannot conclude that these equations are valid for non-zero values of $\epsilon$, i.e., we cannot drop the 0 subscripts in (5.15) and (5.16). It must be emphasized that the exclusion of $A$ from the sum in (5.16) is not arbitrary but rather is a direct consequence of evaluating the integrals in (3.6). In fact it could not be otherwise since all integrals are over surfaces and involve only finite quantities there. It must also be emphasized that the inertial terms in these equations are not inserted by hand by appealing to Newton's laws of motion but are already implicitly contained in (3.6).

\section{B. Post-Coulombian conditions of motion}

To obtain the next, post-Coulombian, conditions on the motion of the sources one needs to evaluate the integrals in (3.6) with $\mu=s$ to an accuracy of $\mathcal{O}\left(\epsilon^{6}\right)$. The Maxwell stress-energy tensor $T^{s r}$ appearing in the integrand can be evaluated to this order from a knowledge of $A_{3}^{r}$ and $A_{4}^{0}$. It follows from the field equations (3.4) and the gauge condition (5.6) that $A_{3}^{r}$ satisfies

$$
\nabla^{2} A_{3}^{r}=0 .
$$

Therefore, as a consequence of the gauge condition, it must have the form

$$
A_{3}^{r}=\sum q_{A} \dot{x}_{A r} / r_{A} .
$$

These same equations and gauge condition also lead to the equation

$$
\nabla^{2} A_{4}^{0}-\ddot{A}_{2}^{0}=0 .
$$

It follows that $A_{4}^{0}$ is given by 


$$
\begin{gathered}
A_{4}^{0}=-\frac{1}{2} \sum q_{A} \ddot{r}_{A} \\
=-\frac{1}{2} \sum q_{A}\left\{\frac{1}{r_{A}}\left[\dot{x}_{A}^{r} n_{A}^{r}\right]^{2}+\ddot{x}_{A}^{r} n_{A}^{r}-\dot{x}_{A}^{2}\right\} .
\end{gathered}
$$

where $n_{A}^{r}=r_{A}^{r} / r_{A}$. No homogeneous solution of equation (5.17) is included here since, by assumption, it would have to be of the form (5.10) and would therefore have the effect of merely redefining the charges $q_{A}$ appearing in these solutions. This expression for $A_{4}^{0}$ appears to contain a term involving the acceleration of the $A$ Th source. However the conditions (5.15) can be used to replace the acceleration factors with Coulomb interaction terms without affecting the overall accuracy of the expression, i.e., it will still be accurate to $\mathcal{O}\left(\epsilon^{4}\right)$.

With these results in hand the evaluation of the second surface integral in (3.6) over a spherical surface surrounding the $A$ Th source to $\mathcal{O}\left(\epsilon^{6}\right)$ is straightforward. Again, one only needs to determine that part of the integral that is independent of $r_{A}$. One finds in this way that

$$
\oint T_{6}^{m s} n_{A \mathrm{~s}} \mathrm{dS} \mathrm{S}_{A}=\frac{2}{3} q_{A} \dot{x}_{A r} F_{3 A}^{m r}-q_{A} F_{4 A}^{m 0}
$$

In this expression $F_{3 A}^{r m}$ and $F_{4 A}^{0 m}$ are again the fields at the location of the $A$ th source produced by all of the other sources. The first of these fields is given by

$$
F_{3 A r s}=\sum^{\prime}\left(A_{3 A s, r}-A_{3 A r, s}\right)=\sum^{\prime} q_{B} \frac{1}{x_{A B}^{3}}\left(\dot{x}_{B}^{r} x_{A B}^{s}-\dot{x}_{B}^{s} x_{A B}^{r}\right) .
$$

Likewise the second field is given by

$$
\begin{gathered}
F_{4 A 0 r}=\sum^{\prime}\left(A_{3 A r, 0}-A_{4 A 0, r}\right) \\
=-\frac{1}{2} \sum^{\prime} q_{B}\left\{\frac{3}{x_{A B}^{5}} x_{A B}^{r}\left(\dot{x}_{B}^{s} x_{A B}^{s}\right)^{2}+\right. \\
+\frac{1}{x_{A B}^{3}}\left(x_{A B}^{r} x_{A B}^{s} \ddot{x}_{B}^{s}-x_{A B}^{r} \dot{x}_{B}^{2}+\frac{1}{x_{A B}} \ddot{x}_{B}^{r}\right\}
\end{gathered}
$$

where again the acceleration factors appearing here are to be replaced by Coulombian interactions with the help of (5.15).

It appears that something is wrong with our calculation, since, among other things we only get $2 / 3$ of the expected Amperian interaction in (5.21) and the sign of the second term on the right hand side of this equations appears to be wrong. We are led to this conclusion however only if we try to interpret equation (3.6) itself as an equation of motion so that we might be led to expect that $\oint U^{\mu r 0} n_{r} d S$ is the 4-momentum of the source contained within the surface over which the integral is performed and $\oint \Theta^{\mu r} n_{r} d S$ is the 4 -force acting on it. In fact they are not. What happens is that part of the first integral contributes the missing 1/3 of the Amperian interaction even though the electromagnetic field does not appear explicitly in its definition. For its evaluation we need to know $U_{5}^{m n 0}$ which, when use is made of the deDonder conditions (5.7), is given by

$$
U_{5}^{m n 0}=\frac{1}{16 \pi}\left(\dot{h}_{4}^{m n}+h_{5}^{m 0}{ }_{, n}\right)
$$

It follows from the field equations (3.1) that $h_{5}^{m 0}$ satisfies

$$
\nabla^{2} h_{5}^{m 0}-\dddot{h}_{3}^{m 0}+4 F_{2}^{r 0} F_{3}^{r m}=0
$$

while $h_{4}^{m n}$ satisfies

$$
\nabla^{2} h_{4}^{m n}-2 \delta^{m n} F_{20 r} F_{2}^{0 r}-4 F_{2}^{0 m} F_{2}^{0 n}=0 .
$$

Thus we see that the electromagnetic field acts, at this order, as a source of the gravitational field and it is these contributions that in turn supply the missing $1 / 3$ of the Amperian force. 
It is not possible to obtain closed form solutions for either of the above equations because of the terms that are quadratic in the fields. However, all that is needed is to determine those parts of the unknown fields $h_{5}^{m 0}$ and $h_{4}^{m n}$ in the neighborhood of a particular source that will yield surface independent terms in order to evaluate the surface integrals surrounding this source. The needed terms are found following procedures developed by EIH and in AI and are given in the appendix. To obtain the post-Coulombian conditions evaluate the surface integrals in (3.6) with $\mu=s$, take the second derivative with respect to $\epsilon$ and set $\epsilon$ equal to zero. The result of these operations yields the conditions

$$
\begin{array}{r}
m_{A 2}\left\{\ddot{x}_{A 2}^{m}+2 \dot{x}_{A 0, t_{3}}^{m}+\dot{x}_{A 0}^{2} \ddot{x}_{A 0}^{m}+\dot{x}_{A 0}^{r} \dot{x}_{A 0}^{m} \ddot{x}_{A 0}^{r}\right\}= \\
q_{A}\left\{\partial_{x_{A B 0}^{r}} F_{2 A 0}^{m 0} x_{A B 2}^{r}+F_{4 A 0}^{m 0}+\frac{1}{2} \dot{x}_{A 0}^{2} F_{2 A}^{m 0}-F_{3 A 0}^{m n} \dot{x}_{A 0}^{n}\right\}
\end{array}
$$

As in all multiple time approximations, the dependence of $x_{A 0}^{m}$ on $t_{3}$ in this equation is determined by the requirement that the totality of the terms that would contribute to the secular growth of $x_{A 2}^{m}$ must vanish.

These conditions, together with the conditions (5.15), are identical in form to the first two slow motion approximations one would obtain from the special relativistic equations of motion for a charged particle of mass $m_{A 2}$ and charge $q_{A}$ moving in the field of a collection of other charges using a multiple time expansion. The second and third terms on the left side of this equation as well as the second term on the right side are equivalent to terms in the special relativistic equations that arise from replacing derivatives with respect to proper time by derivatives with respect to coordinate time and expanding the factors of $1 / \sqrt{1-(\epsilon \dot{x})^{2}}$ that enter as a consequence of the replacement. The first term on the right side is equivalent to a term that is due to the effect of retardation while the last term is the Amperian force. It should be emphasized again however that the conditions derived here differ from the special relativistic equations in a fundamental way in that they are only asymptotic conditions and are not required to be valid at all times. They are also derived from a more fundamental theory without having to be postulated $a b$ initio.

The reader should notice that, without the multiple time formalism, the first two terms on the left side of equation (5.27) would be absent and we would arrive at two totally different equations, (5.15) and (5.27), for the accelerations $\ddot{x}_{A 0}$ and hence a contradiction. In an attempt to avoid this inconsistency some earlier treatments expanded the coordinates $x_{A}$ in a series in $\epsilon$. However, this procedure by itself will fail without the multiple time formalism since secular growth, as in the case of periastron advance, will ultimately lead to a nonuniform expansion.

In arriving at our conditions of motion (5.27) we have consistently ignored surface dependent contributions to our surface integrals since the sum of these contributions must vanish identically. One such contribution does deserve special comment however. In evaluating the surface integral (5.21) one finds a contribution $\frac{2}{3} \ddot{x}_{A}^{m} / r_{A}$. Aside from a difference in the numerical coefficient, this is the same contribution appearing in the line intergral in (2.15) found by Dirac. Here it is exactly canceled by a corresponding term coming from first term in the surface integral in (3.6).

\section{Radiation reaction}

The effects of radiation reaction can be derived using the matched asymptotic expansion method of Burke 20 and its extension to the surface integral method in AI coupled with the method of multiple time scales. Since Burke did not use the surface integral method he had to make an assumption equivalent to assuming the validity of the geodesic equations in order to compute the radiation reaction force. Further, the fact that he did not use multiple time scales meant that his result cannot be considered to be part of a consistent approximation scheme even though he did obtain the "standard" form of the gravitational reaction force.

To determine the electromagnetic reaction force I will proceed as in AI by constructing an outer, slow zone solution and match it to an appropriate inner solution in the neighborhood of a given source. To this end, following Burke, I introduce outer coordinates $\widehat{x}^{\mu}=(\epsilon t, \epsilon \mathbf{r})$. The fields $\widehat{A}^{\mu}(\widehat{x})=A^{\mu}(x)$ are again expanded in what is hopefully an asymptotic series in $\epsilon$ where now the coefficients in the expansion are functions of the outer coordinates. If one imposes the Lorentz gauge condition it then follows from the field equations that, to order $\delta^{2}, \widehat{A}^{\mu}$ satisfies the flat-space homogeneous wave equation

$$
\left(\widehat{\nabla}^{2}-\partial_{\hat{t}}^{2}\right) \widehat{A}^{\mu}=0
$$

Since the inner expansion of the outer solutions of (5.28) must match to the outer expansions of the inner solutions we have already constructed we need these latter expansions. They are obtained by letting $r \rightarrow \infty$ while holding $\epsilon$ fixed. Keeping only the lowest order nontrivial terms needed for this purpose in this limit yields the results 


$$
A_{2}^{0} \rightarrow \frac{Q}{r}+P^{r} \frac{n^{r}}{r^{2}}
$$

where

$$
P^{r}=\sum q_{A} x^{r} A, \quad Q=\sum q_{A},
$$

and

$$
A_{3}^{r} \rightarrow \frac{\dot{P}^{r}}{r}
$$

We see from these outer expansions that the lowest-order contributions to $\widehat{A}^{0}$ must contain terms with zero- and first-order spherical harmonics as factors while those to $\widehat{A}^{r}$ must contain zero-order harmonics.

While the spherical harmonic structure of the outer fields restricts their form it does not fix it - any linear combination of outgoing and incoming wave with the appropriate harmonic dependence will do. In most treatments of radiation it is assumed that only the outgoing wave is present in the wave zone and indeed such was assumed both by Burke and by me in our respective previous works. However, as I later showed, 21] if the energy contained in a wave field on some initial value surface is finite, then in the asymptotic future of this surface the field will be a pure retarded wave. (If one is willing to violate this condition then it is possible to have incoming waves for as long as one wishes into the future.) One could also have solved the wave equation as a final value problem. If one now required that the wave energy on a final value surface be finite then the solution would be a pure advanced wave in the asymptotic past. As a consequence, we see that the choice here of the retarded wave solution to (5.28) is not arbitrary. At the same time it means that any results that depend on this choice such as the about to be derived radiation reaction force will only by valid in the asymptotic future.

Retarded waves are functions of $\widehat{u}=\widehat{t}-\widehat{r}$ and $\widehat{r}$. Consequently the ones that have the right angular dependence are

$$
\widehat{A}^{0}=\frac{b}{\widehat{r}}+\left\{\frac{f_{r}^{\prime}(\widehat{u}, \epsilon)}{\widehat{r}}+\frac{f_{r}(\widehat{u}, \epsilon)}{\widehat{r}^{2}}\right\} n^{r}
$$

and

$$
\widehat{A}^{r}=\frac{g_{r}(\widehat{u}, \epsilon)}{\widehat{r}}
$$

where the prime in equation (5.32) denotes differentiation with respect to $\widehat{u}$. The as yet arbitrary functions $f_{r}(\widehat{u})$ and $g_{r}(\widehat{u})$ appearing in these solutions are not independent. They must be so chosen that the fields $\widehat{A}^{0}$ and $\widehat{A}^{r}$ satisfy the Lorentz gauge condition used in obtaining the wave equation (5.28) from the field equations (3.4). The latter will be the case provided

$$
\mathrm{g}_{r}=f_{r}^{\prime}
$$

To determine these arbitrary functions completely we must match the inner expansions of the outer solutions (5.32) and (5.33) to the outer expansions of the inner solutions (5.29) and (5.31). These outer expansions are obtained by letting $\widehat{r} \rightarrow 0$ while holding $\epsilon$ fixed and are given by

$$
A^{0} \rightarrow \frac{b}{\epsilon r}+\left\{\frac{f_{r}(\epsilon t)}{(\epsilon r)^{2}}-\frac{1}{2} \dddot{f}_{r}(\epsilon t)+\frac{1}{3} \dddot{f}_{r}(\epsilon t) \epsilon r+\cdots\right\} n^{r}
$$

and

$$
A^{r} \rightarrow \frac{\mathrm{g}_{r}(\epsilon t)}{\epsilon r}-\dot{g}_{r}(\epsilon t)+\cdots .
$$

where again a dot over a quantity denotes differentiation with respect to $t_{1}=\epsilon t$. The inner and outer expansion will match if we take

$$
b=\epsilon^{3} Q \quad \text { and } \quad f_{r}(\epsilon t)=\epsilon^{4} P^{r}(\epsilon t) .
$$

In the inner expansion (5.35) the first two terms match to the first two terms in the outer expansion of $A_{2}^{0}$, the third term matches to the first term in the outer expansion of $A_{4}^{0}$ and the first term of the inner expansion (5.36) 
matches to the first term in the outer expansion of $A_{3}^{r}$. However, the time-odd terms in these expansions, the fourth term in (5.35) and the second term in (5.36), do not match to terms in either of these outer expansions. Since both of these terms are solutions of the homogeneous Laplace equation a matching can be achieved by adding solutions of the Laplace equation to our inner solutions whose outer expansions match to these time-odd terms. The required terms are

$$
A_{5}^{0}=\frac{1}{3} \sum q_{A} r_{A}^{r} \dddot{\dddot{x}}_{A}^{r}
$$

and

$$
A_{4}^{r}=\sum q_{A} \ddot{x}_{A}^{r}
$$

The above potentials can now be used to evaluate additional surface independent contributions to 3.6 ) with $\mu=s$. These lowest order contributions to the second integral in this equation are $\mathcal{O}\left(\epsilon^{7}\right)$ and, for a sphere surrounding the Ath source, are given by

$$
\oint_{A} T_{7}^{s r} n_{A}^{r} d S_{A}=\frac{2}{3} q_{A} \sum q_{B} \dddot{x}_{B}^{s} .
$$

Note in particular that in this expression the sum is over all of the sources including the Ath source itself and thus includes, for the first time in these calculations, the effect of a self action. We shall see that this self action is similar to the radiation reaction in the ALD equations.

There is also an $\mathcal{O}\left(\epsilon^{7}\right)$ surface independent contribution to the first integral in equation (3.6) and is given simply by

$$
\left[\oint_{\mathrm{A}} \quad U_{, 0}^{s r 0} n_{A}^{r} d S_{A}\right]_{7}=2 m_{A 0} \partial_{t_{4}} \dot{x}_{A}^{s} .
$$

where $t_{4}=\epsilon^{4} t$. The $\mathcal{O}\left(\epsilon^{7}\right)$ conditions of motion are then gotten by dividing equation (3.6) by $\epsilon^{2}$, differentiating five times with respect to $\epsilon$ and finally setting $\epsilon$ equal to zero. The result of these operations is

$$
2 m_{A 0} \partial_{t_{4}} x_{A 0}^{s}=\frac{2}{3} q_{A} \sum q_{B} \dddot{x}_{B 0 .}^{s}
$$

The right side of (5.42) contains the first time irreversible term in our conditions of motion. Its form and sign is a direct consequence of having solved our wave equations as an initial value problems, leading to the retarded solution in the asymptotic future. If they had been solved as a final value problem so that the solution would have been an advanced wave in the asymptotic past then one would have obtained a result similar to (5.42) but with the sign of the radiation reaction term reversed, thus maintaining the complete symmetry of the basic field equations (3.1) and (3.4). Put another way, the arrow of time points in both directions. Of course, if one were to replace $t$ by $-t$ in the past the advanced solution would transform into a retarded solution and the sign of the reaction term would be reversed.

\section{ALD AND EIH COMPARED}

We have now arrived, starting from the empty-space Einstein-Maxwell equations (3.1), (3.4) and (3.5) and making only the assumption of spherical symmetry for the sources of the gravitational and electromagnetic fields, at a set of constraints (5.15), (5.27) and (5.42) on the motion of these sources. They are applicable only to slowly moving sources and then only in the asymptotic future of some initial value surface. As such, they stand in stark contrast to the ALD equations of motion (2.18) which are held to be exact and to hold for all times. If one were to forget the problems associated with their derivation one would surely favor them over the EIH equations even on esthetic grounds. Except for one problem. The ALD equations admit physically unacceptable solutions - the so-called run-away motions. The EIH conditions on the other hand do not suffer from this defect as we shall see.

The source of the run-away solutions to the ALD equations is of course the third derivative terms in the expression for the radiation reaction force appearing in (2.18). Third derivatives also appear in the radiation reaction term in (5.42). However there is an essential difference in the two cases. In (5.42) the third derivative is with respect to $t_{1}$ and not to the single time variable $t$ appearing in (2.18). The dependence of the source coordinates on $t_{1}$ (and $\left.t_{3}\right)$ is fixed by the Coulombic and post-Coulombic conditions (5.15) and (5.27). Equation (5.42) serves to determine the dependence of the source coordinates on damping time $t_{4}$ and contains only first derivatives with respect to this 
variable. As a consequence, the motions allowed by the EIH conditions are all well behaved. That there are no run-away motions allowed in general relativity follows from the work of Gibbon, Hawking, Horowitz and Perry [25] who have shown that the Bondi mass of a system of charged masses and/or charged black holes is positive definite and the fact that if the Bondi mass is initially finite, its time derivative is negative semi-definite. The Bondi mass thus appears to play the role of a Liapunov function and its existence therefore strongly suggests that these systems are asymptotically stable.

To better understand the fundamental difference between the ALD and EIH equations let us apply them to a simple problem - a non-relativistic oscillator. In this case the ALD equation reduces, for an electron, to

$$
\ddot{x}+\omega_{0}^{2} x=\tau \dddot{x}
$$

where $\tau=\frac{2}{3} e^{2} / m c^{3}$ is the light travel time across the classical radius of the electron. In keeping with the assumption of non-relativistic motion I assume that $\omega_{0} \tau \equiv \epsilon \ll 1$. Finally, introduce a new independent variable $t^{\prime}=\omega_{0} t$ to obtain the equation

$$
\ddot{x}+x=\epsilon \dddot{x}
$$

The solution will be proportional to an exponential $e^{\lambda t}$ where $\lambda$ satisfies

$$
\lambda^{2}+1-\epsilon \lambda^{3}=0
$$

Rather than write out the exact solution for $\lambda$ one can construct a power series solution in $\epsilon$. One finds that

$$
\lambda_{ \pm}= \pm i-\frac{1}{2} \epsilon+\mathcal{O}\left(\epsilon^{2}\right)
$$

There is a third solution given by

$$
\lambda=\frac{1}{\epsilon}+\mathcal{O}(\epsilon)
$$

which is the run-away mode. It has the peculiar feature that $\lambda \rightarrow \infty$ as $\tau \rightarrow 0$, clearly indicating that something is wrong with our equation. Dirac sought to remedy the situation by requiring that the initial acceleration, which must be specified along with the initial position and velocity since we are dealing with a third order equation, should be such as to rule out the run-away solutions. 26. Aside from its ad hoc character, this requirement was shown to lead to acausal behavior. In the present case it would amount to taking the amplitude of the run-away mode to be zero.

Consider now the solution of the same problem using the EIH conditions of motion. In this case (5.15) would have the form

$$
m_{2} \ddot{x}+\omega_{0}^{\prime 2} x=0
$$

where dots now indicate differentiation with respect to $t_{1}$ and a prime is used to differentiate EIH quantities from their corresponding ALD counterparts. Motions allowed by these conditions are of the form

$$
x=A_{ \pm}\left(t_{4}\right) \exp \left( \pm i \omega_{0}^{\prime} t_{1}\right)
$$

where the amplitudes $A_{ \pm}$are now taken to be functions of $t_{4}$. When this result is substituted into (5.42) we obtain the equation

$$
2 i \omega_{0}^{\prime 2} \partial_{t_{4}} A_{ \pm}=-\tau^{\prime} \omega_{0}^{\prime 3} A_{ \pm}
$$

where $\tau^{\prime}$ is the same function of the scaled charge and mass of the electron as $\tau$ is of their unscaled counterparts. With the scalings introduced at the beginning of Section IV we have $\epsilon^{\prime 2} \tau^{\prime}=\tau$. Taking $\epsilon^{\prime} \omega_{0}^{\prime}=\omega_{0}$ we find that

$$
A_{ \pm}=A_{0 \pm} \exp \left(i \tau \omega_{0}^{2} t\right)
$$

Thus we obtain two damped modes corresponding to the two roots 6.4) of (6.3). But we do not obtain a motion corresponding to the run-away mode (6.5) 


\section{SUMMARY}

The conditions of motion derived above together constitute asymptotic conditions on the motion of charged, spherically symmetric, compact, slowly moving sources accurate to $\mathcal{O}\left(\delta^{2}, \epsilon^{7}\right)$. Given the current positions and velocities of a collection of such sources sufficiently far into the future of an initial startup time they will predict their motion for at least a finite time into the future to this order of accuracy. They were derived from the source-free Einstein-Maxwell field equations without additional assumptions concerning force laws or the form of inertial forces. Their derivation did not require assumptions concerning which parts of the fields act on which sources as does the derivation given by Landau and Lifshitz, [19] for example, nor did it require the renormalization of divergent integrals such as in the Dirac derivation. And these conditions do not admit non-physical solutions which violate the approximations used in their derivation. In this connection, Landau and Lifshitz are, to the best of my knowledge, the only authors who suggest that the existence of run-away solutions is evidence for the limited applicability of the radiation reaction force. But then, like other authors, they inexplicably ascribe this state of affairs to the renormalization of the infinite electromagnetic self energy of point sources when in fact no such renormalization is necessary.

We see thus that the outstanding problems of classical electrodynamics, the need for infinite renormalizations and the run-away solutions, find their resolution within the framework of the general theory of relativity. In addition we see how irreversible effects, here radiation damping, arise from reversible fundamental equations. Rather than having to assume an outgoing wave solution in the slow zone valid for all times it is sufficient to assume only that the initial conditions are physically reasonable, that is, that the initial wave energy is finite.

One can ask: is it possible to do better than what has been accomplished here? One can of course go to higher order in $\epsilon$ in the $\mathcal{O}\left(\delta^{2}\right)$ approximation, obtaining thereby higher order special relativistic corrections. One can also go to higher order in $\delta$ to obtain corrections describing electro-gravitational effects.But can one derive equations of motion for these sources rather than conditions of motion? And can one at least derive conditions of motion for arbitrarily moving sources that are special relativistic invariant. The answer to all of these questions is, I believe, most likely no. In the fast zone the motion of the sources will depend in a complicated way not only on their initial positions and velocities but on the initial values of the fields as well. Since during this period there will not in general be any small parameters associated with the motion one would have to solve the field equations exactly in order to evaluate the EIH surface integrals and that, so far, has not been done. It is only if one waits long enough for all of the initial transients in the motion to die down that one can expect to derive relatively simple conditions of motions. For a similar reason one cannot derive special relativistic equations or conditions of motion. All attempts to do so in the past have been singularly unsuccessful, both literally and figuratively. One of course should not feel to badly about this state of affairs since there really does not exist a self-consistent special relativistic description of interacting charged bodies.

To conclude, I want to argue that the derivations of EIH and those here shed an entirely new light on the meaning of equations of motion which lie at the very heart of classical physics. It is not just the fact that conditions of motion can be derived from the empty-space field equations of general relativity that is important but even more so is their very nature. In contrast to equations of motion they are both approximate and asymptotic, they do not hold for all times and they cannot be used to solve initial value problems. This of course does not mean that everything done in the past in classical mechanics is wrong because it used these conditions of motion as equations of motion since their application has always been compatible with the approximations made here. But it does mean, I would also argue, that, in a very real sense, all of classical mechanics and electrodynamics can be looked on as a verification of general relativity since one does not have to postulate the conditions of motion which underlie these theories but can derive them from general relativity.

\section{ACKNOWLEDGMENT}

I would like to thank Joshua N. Goldberg for several very helpful discussions and suggestions for improving the presentation.

\section{APPENDIX:}

In this appendix I will outline the method of determining the field components needed to derive the post-Coulombian conditions of motion (5.27). The first component needed is $h_{4}^{00}$, which satisfies

$$
\nabla^{2} h_{4}^{00}=\ddot{h}_{2}^{00}-2 F_{20 r} F_{20 r} .
$$


This equation can be solved exactly by

$$
\begin{gathered}
h_{4}^{00}=-\left(A_{2}^{0}\right)^{2} \\
-2 \sum m_{A 2}\left[\frac{1}{r_{A}} Y_{A}^{r s} \dot{x}_{A}^{r} \dot{x}_{A}^{s}+Y_{A}^{r} \ddot{x}_{A}^{r}\right]+4 \sum \frac{m_{A 2}}{r_{A}}
\end{gathered}
$$

where

$$
Y_{A}^{r s}=n_{A}^{r} n_{A}^{s}-\frac{1}{3} \delta^{r s} \text { and } Y_{A}^{r}=n_{A}^{r}, \quad n_{A}^{r}=\frac{r_{A}^{r}}{r_{A}}
$$

are spherical harmonics of orders two and one, respectively. The coefficient $m_{A 4}$ in the homogeneous term in the solution (A2) is fixed by (3.6) with $\mu=0$ using the $\mathcal{O}\left(\epsilon^{5}\right)$ terms. The surface independent terms can be made to vanish in this order if we take $\partial_{t_{3}} m_{A 0}=0$, set

$$
m_{A 4}=\frac{1}{2} m_{A 2} \dot{x}_{A 0}^{2}+\frac{1}{2} \sum^{\prime} \frac{q_{B 0}}{x_{A B 0}}
$$

and include the constant of integration in a redefinition of $m_{A 2}$.

The next quantity we need to determine is $h_{4}^{m n}$. Unfortunately, there does not exist a closed form solution of equation (5.26) for these field components. However, all we need to know are those parts of $h_{4}^{m n}$ required for the evaluation of the surface independent terms of the first integral in equation (3.6) with $\mu=s$ over a sphere surrounding the Ath source. These parts can be obtained by expanding the right hand side of equation (5.26) in a Laurent series in $r_{A}$ and keeping those terms that generate these desired parts. One finds in this way that

$$
h_{4 A}^{r s} \simeq 2 m_{A 2}\left\{\delta^{r s} n_{A}^{i} \ddot{x}_{A 0}^{i}-n_{A}^{r} \ddot{x}_{A 0}^{s}-n_{A}^{s} \quad \ddot{x}_{A 0}^{r}\right\}+\frac{b_{A}^{r s}}{r_{A}}
$$

where the $\simeq$ indicates equality modulo terms that do not contribute surface independent terms. The coefficient $b_{A}^{m n}$ in the homogeneous term is fixed by the imposition of the deDonder conditions (5.7) and is given by

$$
b_{A}^{r s}=4 m_{A 2} \dot{x}_{A 0}^{r} \dot{x}_{A 0}^{s}
$$

In a similar manner, the needed parts of $h_{5 A}^{m 0}$ are obtained from equation (5.25) and are found to be given by

$$
\begin{gathered}
h_{5 A}^{0 n} \simeq 2 q_{A} n_{A}^{r} F_{3 A}^{r n}+2 q_{A}\left(\dot{x}_{A 0}^{n} n_{A}^{r}-\dot{x}_{A 0}^{r} n_{A}^{n}\right) F_{2 A}^{r 0}- \\
-2 m_{A 0} \frac{1}{r_{A}}\left(n_{A}^{r} n_{A}^{s}-\frac{1}{3} \delta^{r s}\right) \dot{x}_{A 0}^{r} \dot{x}_{A 0}^{s} \dot{x}_{A}^{n}-2 q_{A} n_{A}^{r}\left(2 x_{A 0}^{\dot{r}} F_{2 A}^{n 0}+\dot{x}_{A 0}^{n} F_{2 A}^{r 0}\right)+ \\
+\frac{f_{A}^{n}}{r_{A}}
\end{gathered}
$$

The last term is a homogeneous term which must again be determined by the imposition of the deDonder coordinate conditions and which in turn requires the knowledge of the $h_{4}^{00}$ found above. When the deDonder conditions are applied one finds that

$$
f_{A}^{n}=\frac{10}{3} m_{A 0} \dot{x}_{A 0}^{2} \dot{x}_{A 0}^{n}
$$

When these expressions for $h_{4 A}^{r s}$ and $h_{5 A}^{m 0}$ are used to evaluate $U_{5 A}^{m n 0}$ and the surface independent terms of the first integral in equation (3.6) together with equation (5.21) one obtains the post-Coulombian conditions (5.27) as described in the text.

[1] J.N. Goldberg, Phys. Rev. 89, 263 (1953). 
[2] A. Einstein, L. Infeld and B. Hoffmann, Ann. Math. 39, 65 (1938).

[3] A. Einstein and L. Infeld, Ann. Math. 41, 455 (1940), Can. J. Math. 3, 209 (1949).

[4] J.L. Anderson, Phys. Rev. D 36, 2301 (1987).

[5] The only exception to my knowledge is P.G. Bergmann, An Introduction to the Theory of Relativity (Prentice-Hall, New York, 1942).

[6] See T. Erber, Fortsch. Phys., 343 (1961) for an extensive discussion and bibliography.

[7] H.A. Lorentz, Theory of Electrons, 2nd edition (1915), (Dover, New York, 1952).

[8] R.P. Feynman, Phys. Rev. 74, 939 (1948).

[9] For the details of the calculation, see S. Coleman, Subnuclear Phenomena, A. Zichichi, ed. (Academic Press, New York, 1970).

[10] J.D. Jackson, Classical Electrodynamics (Wiley, New York, 1962).

[11] F. Rohrlich, Classical Charged Particles (Addison-Wesley, Reading, MA, 1965).

[12] J.L. Jimenez and I. Campos, Am. J. Phys. 55, 1017 (1987).

[13] F.V. Hartemann and N.C. Luhmann, Jr., PR Lett. 74, 1107 (1995) .

[14] P.A.M. Dirac, Proc. Roy. Soc. London, Ser. A 176, 148 (1938).

[15] T.C. Mo and C.H. Papas, Phys. Rev. D 4, 3566 (1971); J.C. Herrera, Phys. Rev. D 15, 453 (1977).

[16] L. Infeld and P.R. Wallace, Phys. Rev. 57, 797 (1940).

[17] For a detailed treatment and history of classical models see E.T. Whittaker, A History of the Theories of Aether and Electricity, 2 vols. (Nelson, London, vol.1, 1951, vol.2 1953; reprinted by Harper, New York, 1960). A summary of the Lorentz derivation can be found in D.J. Jackson, op cit.

[18] For an introduction to these methods see A. Nayfeh, Perturbation Methods (Wiley, New York, 1973).

[19] L.D. Landau and E.M. Lifshitz, The Classical Theory of Fields, (Pergamon, New York, 1971) 3rd revised English ed.

[20] The method of matched asymptotic expansions was first used to derive the gravitational radiation reaction force in W.L. Burke, J. Math. Phys. 12, 401 (1971).

[21] J.L. Anderson, Am. J. Phys. 60, 465 (1992); See also, in this regard, P.C. Aichelburg and R. Beig, Ann. Phys. (N.Y.) 98, 264 (1976).

[22] See, for example, E.A. Frieman, J. Math. Phys. 4, 410 (1963); J.B. Keller and L.L. Bonilla, J. Stat. Phys. 42, 1115 (1986).

[23] For a detailed analysis of length and time scales in gravitationally bound systems, see K.S. Thorne and J.B. Hartle, Phys. Rev. D 31, 1815 (1985).

[24] J.L. Anderson, R.E. Kates, L.S. Kegeles, and R.G. Madonna, Phys. Rev. D 25, 2038 (1982); T. Futamase, Phys. Rev. D 28, 2373 (1983).

[25] G. Gibbon, S. Hawking, G.T. Horowitz and M.J. Perry, Commun. Math. Phys. 88, 295 (1983).

[26] see also in this connection C.J. Eliezer, Rev. Mod. Phys., 19, 147 (1947) and G.N. Plass, Rev. Mod. Phys., 33, 37 (1961). 